\title{
Imagens audiotáteis para estudantes com deficiência visual no ensino de artes: uma revisão bibliográfica sistemática
}

\author{
Audio-tactile graphics for visually impaired students in arts education: \\ a systematic literature review
}

Júlia Pereira Steffen Muniz e Maria Lúcia Leite Ribeiro Okimoto

Imagem audiotátil, ensino inclusivo, ensino de artes, revisão bibliográfica sistemática

Estudantes deficientes visuais ainda enfrentam obstáculos no ambiente de ensino, devido a grande quantidade de imagens presentes em materiais didáticos. Para solucionar esse problema, são utilizadas imagens audiotáteis, já que são recursos que através do design da informação possuem o potencial para conduzir o ensino de artes a um panorama mais inclusivo. Porém, esses recursos não tem seu uso tão difundido no ensino básico, o que foi evidenciado através da realização de uma Revisão Bibliográfica Sistemática (RBS). Dessa forma, o objetivo do artigo é apresentar uma RBS sobre imagens audiotáteis direcionadas ao ensino de arte, e a discussão desses resultados obtidos. Descrevemos os procedimentos para a identificação das lacunas, além de trazer os resultados e reflexões sobre Tecnologia Assistiva, Design Universal e Design Informacional. Como síntese desta revisão se reconhece a importância do papel da imagem audiotátil como elemento de interação para a inclusão de alunos deficientes visuais no ensino de artes, recomendando-se a ampliação da exploração e promoção do tema e sua utilização no ambiente escolar, possibilitando assim um ensino igualitário e acessível.

Audio-tactile graphic, inclusive education, arts education, systematic literature review

Students with visual impairment still face obstacles in teaching environments, due to the large amount of graphics present in teaching materials. To solve this problem, audio-tactile graphics are used, since they are resources that, through information design, have the potential to lead arts education to a more inclusive panorama. However, these resources are not widely used in basic education, which was evidenced by conducting a Systematic Literature Review (SLR). Thus, the objective of this paper is to present a SLR on audio-tactile graphics used in arts education, and the discussion of the results. We describe the procedures for identifying gaps, in addition to bringing the results and reflections on Assistive Technology, Universal Design and Informational Design. As a summary of this review, the importance of the role of the audiotactile graphic as an interaction element for the inclusion of visual impairment students in the teaching of arts is recognized, recommending the expansion of the exploration and promotion of the theme and its use in the school environment, thus enabling an equitable and accessible teaching.

\section{Introdução}

Acredita-se haver no mundo 1 bilhão de pessoas que apresentam algum tipo de deficiência (Nações Unidas, 2016). Já no contexto brasileiro, 23\% da população afirma ser deficiente (IBGE, 2010). No que se refere à deficiência visual, esta é mencionada como a de mais incidência entre os brasileiros, atingindo $77,45 \%$ das pessoas que declaram possuir no mínimo

Anais do $10^{\circ} \mathrm{CIDI}$ e $10^{\circ} \mathrm{CONGIC}$

Kelli C.A.S. Smythe, Rafael de Castro Andrade (orgs.)

Sociedade Brasileira de Design da Informação - SBDI

Curitiba | Brasil | 2021
Proceedings of the $10^{\text {th }} \mathrm{CIDI}$ and $10^{\text {th }}$ CONGIC

Kelli C.A.S. Smythe, Rafael de Castro Andrade (orgs.)

Sociedade Brasileira de Design da Informação - SBDI Curitiba | Brazil | 2021 
uma das deficiências investigadas (deficiência visual, auditiva, motora e mental/intelectual). Em idade escolar (até 17 anos), existem 507 mil estudantes que possuem deficiência visual, apresentando desde alguma dificuldade de enxergar até a cegueira total (IBGE, 2013).

Os dados estatísticos apresentados ressaltam a necessidade de uma sociedade mais inclusiva, em particular na área educativa, que se configura como um dos primeiros e mais importantes meios de socialização e aprendizado. Apesar de avanços em infraestrutura e mobilidade em comparação com décadas anteriores, a realidade dos brasileiros que possuem alguma deficiência e frequentam o ambiente escolar ainda não se demonstra totalmente favorável, já que nem todos os direitos são garantidos de forma prática. Alguns dos principais obstáculos encontrados do ponto de vista do design no ambiente escolar são o mobiliário inadequado dentro da sala de aula; a falta e/ou mal estado das tecnologias assistivas; a insuficiência de material inclusivo que proporcione interação entre pessoas sem e com deficiência visual; a falta de materiais didáticos adequados, como por exemplo, a deficiência de materiais didáticos táteis e com estímulos auditivos (Rocha; Deliberato, 2012; Jacob; Cardoso, 2018; Heidrich; Radai, 2018).

Os materiais didáticos normalmente utilizados de forma mais abrangente nas escolas se utilizam de imagens como forma de transmissão do conhecimento, dificultando muitas vezes a absorção dos conteúdos por alunos com deficiência visual. Nesse contexto, o ensino de artes aborda em quase sua totalidade as temáticas de forma visual, o que acaba acentuando a exclusão do aluno com deficiência visual dentro do ambiente escolar (Silva et al., 2010). Em muitas ocasiões, a descrição verbal da imagem realizada pelo professor é a única maneira da pessoa com deficiência visual ter acesso às imagens que são expostas (Posca, 2017; Álvarez, 2003).

Dessa forma, as imagens audiotáteis se configuram como um meio de permitir o acesso de estudantes com deficiência visual a imagens utilizadas durante o processo de ensinoaprendizagem, onde através da percepção tátil (pontos, linhas, áreas, volumes e textos em Braille) e auditiva (audiodescrições, sons e músicas), o estudante explora e obtém as informações do conteúdo. Como um objeto de aprendizagem, já que possui um objetivo de aprendizagem definido, as imagens audiotáteis buscam proporcionar às pessoas com deficiência visual uma informação específica (Macedo, 2010).

Assim, o Design da Informação se configura como um meio para transformar a imagem bidimensional presente em materiais didáticos, em uma imagem audiotátil. Apesar de o conceito de Design da Informação não possuir apenas uma definição correta, de acordo com Junior, Castillo e Coutinho (2015), compartilham um ponto em comum, sendo ele a manipulação e preocupação em passar para o usuário final uma mensagem de maneira eficaz e eficiente, satisfazendo suas necessidades informacionais. Portanto, mesmo que usualmente o Design da Informação esteja mais relacionado à parte gráfica, ele também pode se utilizar de outros sentidos na transmissão do conhecimento, como é o caso do tato e da audição (Junior, Castillo e Coutinho, 2015). 
Sendo assim, o objetivo deste artigo é apresentar uma Revisão Bibliográfica Sistemática sobre imagens audiotáteis direcionadas ao ensino de arte e a discussão desses resultados obtidos, trazendo lacunas e reflexões sobre o tema.

\section{Referencial Teórico}

A deficiência visual pode ser dividida entre a perda total da visão (cegueira) ou a perda parcial da visão (baixa visão), não sendo possível a correção por meio de lentes (Santos e Silva, 2013). Assim sendo, o termo correto para denominar alguém que possui a perda total ou parcial da visão é pessoa com deficiência visual, já que o Brasil ratificou com valor de emenda constitucional em 2008 o termo presente na Convenção sobre os Direitos das Pessoas com Deficiência (PMC, 2013). Para que a educação de pessoas com deficiência visual seja realizada de forma adequada, com a garantia de seus direitos como cidadãos, é indispensável que sejam educados a partir de uma perspectiva inclusiva. Autores como Quevedo e Ulbricht (2011) indicam que com a falta do ensino inclusivo, é possível que o estudante com deficiência visual desenvolva atrasos motores e cognitivos, onde tais problemas não ocorrem como uma consequência da deficiência, mas sim pela falta de experiências do indivíduo com o seu entorno e contexto.

Dessa forma a Tecnologia Assistiva (TA), desenvolvida através do Design Universal, contribui para que a inclusão no ambiente escolar seja alcançada pelo maior número de alunos possível. A TA se configura como uma área do conhecimento interdisciplinar que "engloba produtos, recursos, metodologias, estratégias, práticas e serviços que objetivam promover a funcionalidade, relacionada à atividade e participação, de pessoas com deficiência, incapacidades ou mobilidade reduzida, visando sua autonomia, independência, qualidade de vida e inclusão social" (Brasil, 2009, p. 30). No que se refere especificamente à esfera educacional, a TA caracteriza-se como um conjunto de recursos que possui como objetivo promover o acesso e a participação dos alunos deficientes visuais na aprendizagem (Brendler et al., 2014). Assim sendo, a TA busca proporcionar uma ampliação da comunicação no aprendizado da pessoa com deficiência visual (Bersch, 2013).

Para que esses recursos sejam elaborados de forma que contemplem o maior número de usuários possíveis, o Design Universal deve ser utilizado na concepção de produtos, ambientes, programas e serviços (Gabrilli, 2016). Assim, proporciona o acesso à informação de diferentes públicos, democratizando o processo de ensino-aprendizagem.

O Design da Informação nessa conjuntura têm a capacidade de modificar o cenário de exclusão dentro de sala de aula, proporcionando a inclusão, já que procura aperfeiçoar o processo de aquisição de informações, promovendo a eficiência comunicativa (Junior, Castillo e Coutinho, 2015). Por não se limitar ao aspecto visual, é recomendado que sejam usados no ato de projetar informações pictóricas, acústicas, táteis e/ou olfativas, moldando assim a estrutura da informação que se planeja transmitir (IID, 2007). 
Nesse contexto, a imagem audiotátil se utiliza de informações táteis e auditivas para transmitir as informações necessárias para principalmente, pessoas com deficiência visual. Apesar de serem similares às imagens táteis, contam com o acréscimo de informações sonoras de forma simultânea, acarretando em um melhor aprendizado, já que não existe o sobrecarregamento de informações em apenas um sentido. Esse excesso de informações se dá pelo fato de que o estudante com deficiência visual utiliza o tato tanto para adquirir informações textuais, através do Braille, como também para informações gráficas (Muniz, Sanches e Okimoto, no prelo). Assim, Rossetti et al. (2018) e Götzelmann (2018), comentam que a junção dos estímulos auditivos e táteis no mesmo artefato ocasiona a diminuição do esforço cognitivo empregado na compreensão, auxiliando de uma forma mais precisa a formação de imagens mentais do objeto real.

Como forma de produção, destaca-se a impressão 3D. De acordo com Júnior, Castillo e Coutinho (2015, p. 1356), a impressão 3D pode auxiliar o design de informação ao "criar elementos informacionais que explorem, além da visão, outros canais sensoriais, estimulando a percepção e a cognição na construção do conhecimento". O processo possui um grande potencial como um recurso assistivo, tendo o objetivo de tornar o acesso à informação mais acessível (Sanches, 2018; Júnior, Castillo e Coutinho, 2015).

Como as imagens audiotáteis possuem a capacidade de transmitir conhecimentos que normalmente são encontrados em imagens bidimensionais dentro de recursos didáticos, possuem um grande escopo de utilização dentro do ensino de arte. Em uma análise aos documentos dos Parâmetros Curriculares Nacionais (PCNs), é possível verificar que tanto no Ensino Fundamental como no Ensino Médio, é esperado que o estudante desenvolva capacidades relacionadas à observação de obras de artistas (Brasil, 1997; Brasil, 1998; Brasil, 2000).

No Ensino Médio, por exemplo, destaca-se o campo de competências denominado "Apreciar produtos de arte, em suas várias linguagens, desenvolvendo tanto a fruição quanto a análise estética, conhecendo, analisando, refletindo e compreendendo critérios culturalmente construídos e embasados em conhecimentos afins, de caráter filosófico, histórico, sociológico, antropológico, psicológico, semiótico, científico e tecnológico, dentre outros" (Brasil, 2000, p. 53). Dentro desse bloco, espera-se que os alunos adquiram as seguintes capacidades:

- Fruir, estudar e analisar as produções em artes visuais, tanto na produção artística em geral quanto naquelas ligadas ao campo da comunicação visual como o designer, ou ainda naqueles produzidos pelas novas mídias e artes audiovisuais - vídeo, televisão, multimídia, CD-ROM, home-page etc - conscientizando-se dos meios visuais e audiovisuais de representação, comunicação e informação;

- Investigar as articulações dos elementos e componentes básicos das linguagens visual e audiovisual presentes nas produções artísticas em geral e nas do campo da comunicação visual, das novas mídias e artes audiovisuais. 
Consequentemente, o aluno com deficiência visual pode ser extremamente afetado nesse contexto, já que é através da leitura de obras de arte que o aluno é preparado para a decodificação da imagem física, preparando-se assim para a assimilação da imagem de uma forma geral (Barbosa, 2019). É através do ensino de arte em instituições públicas, que o acesso à arte se torna possível para a maioria dos estudantes no país, exercendo assim o princípio democrático de acesso a informação (Barbosa, 2019).

Dessa forma, é possível que o estudante com deficiência visual não manifeste interesse durante a disciplina de artes por não entender de forma efetiva o conteúdo que é transmitido por meio do ensino-aprendizagem, como por exemplo, em questões relativas às obras de arte como perspectiva, volume, textura, entre outros (Muniz, Sanches e Okimoto, 2021). Assim, acredita-se que seja necessário oferecer soluções que tragam estímulos por outros canais sensoriais além da visão, como é o caso dos estímulos táteis e auditivos.

A partir desses pressupostos, buscou-se validar a existência de uma lacuna na utilização de imagens audiotáteis no ensino de arte, através de uma RBS, e assim também conhecer o estado da arte.

\section{Método}

A Revisão Bibliográfica Sistemática se caracteriza por ser um método de revisão em que é possível fazer sua replicabilidade, através da explicitação do processo como foi conduzida, permitindo a rastreabilidade dos critérios adotados ao longo de sua realização (Santos, 2018). Assim, permite de maneira geral a reconstrução coerente e integrada do corpo técnico associado a um determinado problema; identificação de lacunas, contradições ou convergências dentro de uma temática de investigação e a formulação de uma pergunta de investigação científica relevante para a sociedade e para o avanço do campo de conhecimento.

\section{Revisão Bibliográfica Sistemática}

Para o desenvolvimento desta RBS, foi realizada uma adaptação do roteiro proposto por Conforto e Silva (2011), onde o método está dividido em três etapas: Entrada, Processamento e Saída. Inicialmente foi definido o objetivo, as fontes primárias, as palavras-chave e os critérios de inclusão e exclusão. Seguidamente foi realizada na etapa de Processamento a condução das buscas e a análise dos resultados obtidos. Por fim, na etapa de Saída, foi feito o cadastro e arquivo dos documentos, assim como a síntese dos resultados.

\section{Entrada}

A etapa de Entrada caracteriza-se como a parte inicial, onde se definem o objetivo, fontes primárias, palavras-chave e os critérios de inclusão e exclusão. O(s) objetivo(s) da RBS deve estar alinhado com os objetivos do projeto de pesquisa, já que será a base para a análise dos documentos encontrados na busca (Conforto e Silva, 2011). Nesse caso, o objetivo da RBS é 
definir se existe a lacuna de utilização de imagens audiotáteis no ensino de arte durante o ensino básico por alunos com deficiência visual, fabricadas principalmente com a impressão 3D.

Posteriormente foram escolhidas as fontes primárias, onde foi feita a identificação e seleção dos bancos de dados, de acordo com disponibilidade dos arquivos de forma de integral e gratuita proporcionada pela Universidade Federal do Paraná (UFPR) e pela abrangência do tema pesquisado dentro de cada banco. A partir disso, os bancos de dados selecionados foram o Periódico CAPES, Teses e Dissertações UFPR, Google Acadêmico, Web of Science e Scopus.

Também foram selecionadas as palavras-chave, que nesse caso, foram escolhidas a partir do estudo preliminar de fontes e por uma consulta realizada com pesquisadores. As palavraschave foram definidas a partir do mais abrangente, com o objetivo de encontrar as lacunas possivveis para atuação. Foram selecionadas duas palavras-chave principais (deficiência visual e cego), relacionadas aos usuários, que foram combinadas com mais cinco palavras-chave secundárias (educação inclusiva, tecnologia assistiva, manufatura aditiva, impressão 3D e objeto educacional), tanto em português como em inglês, totalizando 20 combinações diferentes (Tabela 1).

Tabela 1: Palavras-chave selecionadas

\begin{tabular}{ll}
\hline Palavras-chave em português & Palavras-chave em inglês \\
\hline Deficiência visual & Visual Impairment \\
Cego & Blind \\
Educação Inclusiva & Inclusive Education \\
Tecnologia Assistiva & Assistive Technology \\
Manufatura Aditiva & Addtive Manufacture \\
Impressão 3D & 3D Printing \\
Objeto Educacional & Educational Object \\
\hline
\end{tabular}

Fonte: as autoras (2021)

Por fim, foram selecionados os critérios de inclusão e exclusão, que foram definidos de acordo com o objetivo da RBS. A partir do objetivo de validar uma lacuna existente, os critérios de inclusão e exclusão foram estabelecidos para selecionar a produção recente sobre o tema, focando-se em imagens audiotáteis dirigidas a pessoas com deficiência visual (Tabela 2). 
Tabela 2: Critérios de Inclusão e Exclusão

\section{Critérios de Inclusão e Exclusão}

Critérios de Inclusão Documentos onde a pesquisa foi dirigida ao ensino básico (Ensino

Fundamental e Ensino Médio), independente da área de atuação

Documentos que tratem de imagens audiotáteis aplicadas no meio artístico, independente do contexto aplicado

Critérios de Exclusão Documentos produzidos anteriormente de 2010

Periódicos não revisados por pares, no caso de artigos

Documentos sobre metodologias aplicadas aos professores

Documentos disponíveis de forma incompleta (descartados resumos, resumos expandidos e resenhas)

Documentos onde a única adaptação realizada para pessoas com deficiência visual foi a utilização da escrita em formato Braille

Documento onde a pesquisa foi aplicada com outros tipos de deficiências

Fonte: as autoras (2021)

Com o estabelecimento dos critérios de inclusão e exclusão, foi iniciada a etapa de Processamento.

\section{Processamento}

A etapa de Processamento foi dividida em três fases: Condução das Buscas, Análise dos Resultados e Documentação. Para iniciar as buscas, foram utilizados os filtros definidos por Conforto e Silva (2011), aplicados sucessivamente:

- Filtro 1: leitura do título, resumo e palavras-chave;

- Filtro 2: leitura da introdução e conclusão;

- Filtro 3: leitura completa do documento.

Em algumas bases de dados foi necessário aplicar outros filtros para selecionar os temas pesquisados, com o objetivo de diminuir e selecionar os resultados de acordo com o objetivo da pesquisa, que estão descritos no tópico seguinte em conjunto com os resultados obtidos.

Posteriormente, foi iniciada a Análise dos Resultados. Para uma melhor visualização e síntese, os resultados obtidos serão descritos abaixo de acordo com os bancos de dados pesquisados, citando também a análise da bibliografia realizada.

- Portal de Periódicos Capes: devido ao grande número de resultados no idioma inglês, foram aplicados filtros específicos de exclusão com o objetivo de diminuir e especificar de maneira mais produtiva para a pesquisa os artigos selecionados, antes de serem gerados os resultados iniciais. Dessa forma, só foram considerados temas relativos à educação. Após a aplicação do Filtro 3 , foram classificados 10 documentos. 
Posteriormente, foi feita a análise da bibliografia dos 10 artigos selecionados para possivelmente, ampliar o escopo de publicações sobre o tema em questão. Foram utilizados os mesmos critérios de inclusão e exclusão definidos anteriormente. Apesar disso, não foi possível encontrar outras referências que se enquadram no escopo da pesquisa.

- Teses e Dissertações UFPR: após o terceiro filtro, foram selecionados três documentos pertinentes à pesquisa. Posteriormente, foi feita a análise da bibliografia dos três artigos selecionados, onde foram encontrados mais quatro documentos relevantes.

- Google Acadêmico: devido ao grande número de resultados obtidos, foram aplicados os filtros apenas nos 200 primeiros artigos selecionados por ordem de relevância. Dessa forma, foram classificados 19 documentos após a aplicação do terceiro filtro. Posteriormente, foi efetuada a análise da bibliografia dos documentos, onde foi selecionado mais um artigo pertinente ao objetivo de pesquisa.

- Web of Science: foi classificado após o filtro 3 apenas um documento relevante à pesquisa. Após a classificação do artigo, foi efetuada a análise bibliográfica, que não demonstrou resultados pertinentes.

- Scopus: em decorrência do número de documentos a serem analisados em algumas combinações de palavras no idioma inglês, foram aplicados filtros específicos de exclusão com o propósito de maximizar os resultados que possuem conexão direta ao objetivo da RBS, considerando assim apenas temas pertinentes à educação. Ao final dos três filtros, foram classificados dois documentos. Logo após, foi feita a análise da bibliografia dos três artigos selecionados, onde não foram obtidos resultados relevantes para a RBS.

Em seguida, foram compiladas todas as informações obtidas no processo e sintetizadas em formas de quadros e tabelas, para uma melhor síntese visual, totalizando 41 artigos classificados. A tabela 3 demonstra o processo de constrição de toda a RBS, podendo assim ser mantido um comparativo entre as bases de dados e os respectivos resultados.

Tabela 3: Processo de Constrição Final

\begin{tabular}{|c|c|c|c|c|}
\hline Base de Dados & $\begin{array}{l}\text { Resultados } \\
\text { Totais }\end{array}$ & $\begin{array}{l}\text { Após Critérios } \\
\text { e Filtros }\end{array}$ & $\begin{array}{l}\text { Classificados pela análise } \\
\text { de bibliografia }\end{array}$ & $\begin{array}{l}\text { Total de } \\
\text { Classificados }\end{array}$ \\
\hline Periódico Capes & 61.537 & 10 & 00 & 10 \\
\hline $\begin{array}{l}\text { Teses e } \\
\text { Dissertações } \\
\text { UFPR }\end{array}$ & 3.777 & 03 & 04 & 07 \\
\hline $\begin{array}{l}\text { Google } \\
\text { Acadêmico }\end{array}$ & 1.704 .515 & 19 & 01 & 20 \\
\hline Web of Science & 1.724 & 01 & 00 & 01 \\
\hline Scopus & 32.297 & 03 & 00 & 03 \\
\hline Total & 1.803 .850 & 36 & 05 & 41 \\
\hline
\end{tabular}

Fonte: as autoras (2021) 
Após a obtenção dos documentos selecionados, foi iniciada a etapa de Saída.

\section{Saída}

A última etapa do roteiro da RBS é denominada Saída, composta pelo cadastro e arquivo dos documentos e a síntese dos resultados obtidos. De acordo com o modelo proposto por Conforto e Silva (2011), os artigos que forem selecionados depois da aplicação do terceiro filtro, devem ser analisados e interpretados e posteriormente devem ser incluídos no repositório de artigos da pesquisa. Assim, foi feito o dowload de todos os documentos em formato completo, sendo separados por níveis de proximidade com a pesquisa. Uma descrição mais completa das diferenciações e proximidades está descrita na seção "Resultados e Discussão", juntamente com a fase de síntese dos resultados obtidos.

\section{Resultados e Discussão}

De acordo com o objetivo proposto pela RBS, de validar a existência de uma lacuna informacional, a análise dos resultados foi focada para esse propósito. Como exposto no referencial teórico, compreende-se que as imagens audiotáteis são materiais insuficientemente explorados na educação, mesmo tendo um notável potencial de ensino ao serem utilizadas na inclusão do estudante com deficiência visual durante o ensino de arte. Fato esse, pôde ser confirmado através da RBS, onde é notada a discrepância entre o uso de materiais didáticos táteis e materiais audiotáteis. Do total de 41 artigos selecionados, apenas três citam o uso de estímulos auditivos e táteis em um único material didático $(7,3 \%)$, e nenhum deles se concentra na área artística.

Também é perceptível nos documentos selecionados, que a maioria dos recursos didáticos são direcionados ao âmbito da geografia, representada pelos mapas táteis aplicados em diversos contextos. Em seguida, se encontra a área artística como um todo (museus e ensino), e posteriormente as áreas de matemática e história com um total de três documentos cada uma, entre outros com menor porcentagem (Tabela 4).

Tabela 3: Resultados da RBS por área de conhecimento

\begin{tabular}{lll}
\hline Áreas de Conhecimento & $\begin{array}{l}\text { Quantidade de Documentos em } \\
\text { relação ao total }\end{array}$ & $\begin{array}{l}\text { Porcentagem em relação ao } \\
\text { total }\end{array}$ \\
\hline Geografia & 16 & $39,03 \%$ \\
Química & 02 & $4,88 \%$ \\
Biologia & 02 & $4,88 \%$ \\
História & 03 & $7,32 \%$ \\
Matemática & 03 & $7,32 \%$ \\
Ciências & 01 & $2,43 \%$ \\
Física & 01 & $2,43 \%$ \\
\hline
\end{tabular}




\begin{tabular}{lll}
\hline Artes & $\mathbf{0 9}$ & $\mathbf{2 1 , 9 5 \%}$ \\
Astronomia & 02 & $4,88 \%$ \\
Outros & 02 & $4,88 \%$ \\
\hline
\end{tabular}

Fonte: as autoras (2021)

Dentre a porcentagem relativa à área artística, a grande maioria está relacionada à acessibilidade em museus (92,7\%), e apenas 7,3\% são de aplicações dentro do ensino. Esse fato pode estar relacionado com a falta de investimentos em recursos didáticos para alunos deficientes, já que em contrapartida, muitos museus são privados e podem ter mais investimentos em acessibilidade por conter um orçamento maior. De modo geral, apenas $24 \%$ das imagens audiotáteis foram aplicadas dentro do ensino básico (brasileiro ou internacional), $\mathrm{o}$ que ajuda a corroborar essa conclusão.

Também foi realizada uma análise sobre o modo de fabricação, buscando entender possíveis lacunas, diretrizes e/ou sugestões para a confecção de modelos tridimensionais. Apesar de algumas iniciativas, como por exemplo, as 27 materializações realizadas com impressão 3D identificadas nos documentos selecionados pela RBS ( $66 \%$ dos documentos), muitos desses materiais didáticos são feitos de forma isolada. Além disso, muitos são desenvolvidos com materiais que apesar de possuírem um preço acessível, apresentam uma baixa durabilidade como, por exemplo, papéis em geral, E.V.A. e biscuit. Ressalta-se que apesar do número de imagens e modelos produzidos por impressão 3D e por outros materiais nos documentos, muitas produções realizadas no âmbito bidimensional não foram classificadas pela RBS, o que demonstra que existem numerosos objetos de aprendizagens que se utilizam de materiais que acabam proporcionando um pequeno tempo de uso, devido a sua baixa durabilidade e alto desgaste. Dessa forma, acredita-se que a impressão 3D seja de grande utilidade na produção e confecção de recursos didáticos, e concorda-se com Júnior, Castillo e Coutinho (2015) e Sanches (2018), no que se refere ao potencial assistivo que a impressão 3D possui dentro da questão da educação inclusiva.

Dentre todos os documentos selecionados, apenas um deles apresenta a relação entre o ensino básico e o ensino de arte utilizando-se da impressão 3D como meio de fabricação. Moreira, Periotto e Tenório (2020) realizaram um estudo através de um estudo de caso na cidade de Terra Boa - PR, no Colégio Estadual Helena Kolody. Dentro de um projeto da própria escola, intitulado "Laboratório Criativo", os autores do artigo em conjunto com mais cinco alunos, entre ele um aluno com deficiência visual do Ensino Médio, reproduziram obras de arte, para que estas pudessem ser lidas pelo aluno cego que fazia parte do grupo.

Evidencia-se o interesse e a empolgação dos alunos videntes em produzir o recurso didático que possibilitasse ao aluno com deficiência visual a sua inclusão em sala de aula, proporcionando assim que esse material fosse acessivel a todos os estudantes dentro daquele espaço em específico.

Para que isso aconteça, ressalta-se a necessidade do uso do Design Universal na produção de materiais didáticos assistivos, já que esses objetos de ensino facilitarão o bom desempenho 
do aluno no processo de ensino-aprendizagem, além de promover a autonomia e a independência dos estudantes e proporcionar o uso desse objeto para o maior número de pessoas possível.

\section{Considerações Finais}

Através da realização da RBS, foi possível confirmar a existência de uma lacuna informacional, o que é de extrema importância para justificar uma pesquisa acadêmica, já que é necessário poder contribuir de maneira palpável para a área do conhecimento do Design Informacional e para a acessibilidade no ensino de artes. Com isso, é altamente recomendável o uso da RBS como um método para validar lacunas existentes no campo do Design como um todo.

No que se refere à inclusão, ainda existe um caminho a ser percorrido. É notável a necessidade da criação de materiais acessíveis no ensino de arte, já que é possível que o aluno não demonstre interesse na disciplina ou não tenha um bom rendimento por não entender de maneira satisfatória sobre o conteúdo que é tratado em sala de aula. Por isso, precisam existir mais iniciativas que promovam a utilização de imagens audiotáteis dentro do ambiente escolar, visando um custo relativamente baixo, para que seja possível a democratização do ensino através dos materiais didáticos.

Considera-se assim que a impressão 3D seja uma ferramenta que pode contribuir para essa democratização do ensino, além de propiciar a acessibilidade em projetos que incluam o design da informação. Dessa forma, o design da informação se configura como algo de extrema importância no momento projetual de uma imagem audiotátil, já que é através dele que as informações proporcionarão uma mensagem eficaz e eficiente ao aluno.

\section{Agradecimento}

Agradecimentos a CAPES pela bolsa de mestrado concedida, possibilitando assim a viabilização da pesquisa.

\section{Referências}

Álvarez, J. A. B. (2003). Multissensorialidade no ensino de desenho a cegos. São Paulo.

Barbosa, A. M. (2019). Arte-educação no Brasil. São Paulo: Editora Perspectiva.

Bersch, R. (2013). Introdução à tecnologia assistiva. Porto Alegre. Disponível em https://www.assistiva.com.br/Introducao_Tecnologia_Assistiva.pdf.

Brasil. (2009). Tecnologia Assistiva. Subsecretaria Nacional de Promoção dos Direitos da Pessoa com Deficiência, Comitê de Ajudas Técnicas. Brasília: CORDE.

Brasil (1997). Parâmetros curriculares nacionais: artes. Secretaria de Educação Fundamental. Brasília: MEC/SEF.

Brasil (1998). Parâmetros curriculares nacionais: artes. Secretaria de Educação Fundamental. Brasília: MEC/SEF.

Brasil (2000). Parâmetros curriculares nacionais (Ensino Médio). Brasília: MEC. 
Brendler, C. F., Viaro, F. S., Bruno, F. B., Teixeira, F. G. Silva, R. P. (2014). Recursos didáticos táteis para auxiliar a aprendizagem de deficientes visuais. Educação Gráfica. 18.

Conforto, E. C. \& Silva, S. L. (2011). Roteiro para Revisão Bibliográfica Sistemática: aplicação no desenvolvimento de produtos e gerenciamento de projetos. Porto Alegre, RS.

Gabrilli, M. (2016). Guia sobre a Lei Brasileira de Inclusão - LBI. Disponível em http://maragabrilli.com.br/wp-content/uploads/2016/03/Guiasobre-a-LBI-digital.pdf.

Götzelmann, T. (2018). Visually augmented áudio-tactile graphics for visually impaired people. ACM Transactions on Accessible Computing. 11 (2).

Heidrich, R. O. \&. Radai, K. S. (2018). Tabuleiro da geografia: protótipo de jogo para crianças deficientes visuais. $13^{\circ}$ Congresso Brasileiro de Pesquisa e Desenvolvimento em Design.

Jacob, E. M. \& Cardoso, F.A. (2018). Vamos brincar? Material lúdico-pedagógico voltado para crianças cegas e de baixa visão em fase de escolarização. Joinville, SC.

Junior, N. C., Castillo, L. G., Coutinho, S. G. (2015). A impressão 3D contribuindo em projetos de design de informação. 7 th Information Design Internacional Conference. 2 (2). São Paulo: Blucher.

IBGE - Instituto Brasileiro de Geografia e Estatística. (2010). Censo demográfico 2010: características gerais da população, religião e pessoas com deficiência. Disponível em http://biblioteca.ibge.gov.br/visualizacao/periodicos/94/cd_2010_religiao_deficiencia.pdf.

IBGE - Instituto Brasileiro de Geografia e Estatística (2013). Pesquisa Nacional de Saúde: acesso e utilização dos serviços de saúde, acidentes e violências. Coordenação de Trabalho e Rendimento. Rio de Janeiro.

IIID. (2007). International Institute for Information Design. Disponível em https://www.iiid.net/ PDFs/idxPublication.pdf.

Macedo, C. M. S. (2010). Diretrizes para criação de objetos de aprendizagem acessíveis. Tese de Doutorado. Universidade Federal de Santa Catarina, Florianópolis, SC.

Moreira, G. R., Periotto, T. C.. Tenório, N. (2020). Criação e compartilhamento do conhecimento usando tecnologia de impressão 3D em obras de arte para o aluno deficiente visual. Brazilian Journal of Development. Curitiba, PR.

Muniz, J. P. S, Sanches, E. C. P., Okimoto, M. L. L. R. (no prelo) Imagens audiotáteis no ensino de artes para estudantes cegos: lacunas e reflexões.

Nações Unidas (2016). The invisibility of disability. Disponível em http://www.un.org/ disabilities/documents/sdgs/infographic_statistics_2016.pdf.

Posca, L. M. (2017). Criação de material de apoio para o ensino básico de artes visuais para alunos deficientes visuais. Uberlândia, MG.

PMC - Prefeitura Municipal de Curitiba (2013). Terminologia sobre a pessoa que têm deficiência. Curitiba, PR.

Quevedo, S. R. P. \& Ulbricht, V. R. (2011). Como os cegos aprendem. Florianópolis, SC.

Rocha, A. N. D. C. \& Deliberato, D. (2012). Tecnologia assistiva para criança com paralisia cerebral na escola: identificação das necessidades. Marília, SP. 
Rossetti, V., Furafari, F., Leporini, B., Pelagatti, S., Quarta, A. (2018). Smart Cultural Site: an interactive $3 \mathrm{~d}$ model acessible to people with visual impairment. IOP Conference Series: Materials Science and Engineering. 364 (1).

Sanches, E. C. P. (2018). Modelo de tradução para acessibilidade de imagens estáticas de objetos de aprendizagem através de impressão tridimensional. Dissertação de Mestrado. Universidade Federal do Paraná, Curitiba, PR.

Santos, A. (2018). Seleção do método de pesquisa: guia para pós-graduando em design e áreas afins. Curitiba, PR.

Santos, W. C. \& Silva, R. S. (2013). Auxílio ao processo de inclusão de alunos com deficiência visual como condição para uma aprendizagem de qualidade. 29 (4).

Silva, K. C., Santiago, J. V. B., Dickman, A. G., Ferreira, A. C. Auxiliando o ensino de química orgânica para alunos com deficiência visual: materialização de compostos moleculares. $P B L$ 2010 Congresso Internacional. São Paulo, SP.

\section{Sobre o(a/s) autor(a/es)}

Júlia Pereira Steffen Muniz, Mestranda, UFPR, Brasil <juliasteffenmuniz@hotmail.com> Maria Lúcia Leite Ribeiro Okimoto, Doutora, UFPR, Brasil <lucia.demec@ufpr.br> 